\title{
PRÉ-NATAL NA ATENÇÃO BÁSICA: CONHECIMENTOS MÉDICOS
}

\author{
PRENATAL CARE IN PRIMARY HEALTH CARE: MEDICAL KNOWLEDGE
}

\author{
PRENATAL EN LA ATENCIÓN BÁSICA: CONOCIMIENTOS MÉDICOS
}

\begin{abstract}
Adriano Antonio da Silva Pedrosa ${ }^{1}$, Maria das Graças Monte Mello Taveira ${ }^{2}$,́talo de Barros Freitas $^{3}$, Guilherme Monteiro Constant ${ }^{4}$, DivaniseSuruagy Correia ${ }^{5}$, Jairo Calado Cavalcante $^{6}$
\end{abstract}

\section{RESUMO}

Objetivo: avaliar o conhecimento sobre pré-natal na Atenção Básica $(A B)$ entre médicos que atuam no Programa Mais Médicos para o Brasil (PMMB). Método: trata-se de um estudo quantitativo, transversal e analítico realizado com 101 médicos do PMMB em Alagoas. O estudo foi realizado em Maceió, em amostra por conveniência não probabilística, em 2017, no momento do encontro locorregional do PMMB. Aplicou-se um questionário composto por dez perguntas de múltipla escolha abordando o tema do prénatal na $A B$.Os dados foram analisados no programa Epi Info por meio de medidas estatísticas de frequência e qui-quadrado. As questões foram divididas por temas: duas abordando Diabetes Mellitus Gestacional; duas sobre morbimortalidade materna; três a respeito de exames de imagem em obstetrícia e vitalidade fetal e três sobre exames complementares no pré-natal. Resultados: dos 101 médicos, 33,3\% eram brasileiros e $66,7 \%$, estrangeiros; $62,7 \%$ eram mulheres. Os profissionais brasileiros obtiveram uma média de acertos superior em relação aos estrangeiros, $61,67 \%$ contra $42,53 \%$, sendo a média geral $49 \%$. Conclusão: isso pode sugerir uma discrepância no direcionamento dos modelos educacionais, com um melhor enfoque no pré-natal por parte das faculdades brasileiras.

\section{Descritores: Cuidado Pré-Natal; Educação Continuada; Atenção Primária à Saúde; Médicos.}

\section{ABSTRACT}

Objective: to evaluate the knowledge about prenatal care in Primary Care (PC) among doctors who work in the MaisMédicos para oBrasil Program (PMMB). Method: it is a

\footnotetext{
1,6 Médico. Universidade Federal de Alagoas/UFAL. Maceió (AL), Brazil.

${ }^{2}$ Mestra. Universidade Federal de Alagoas/UFAL. Maceió (AL), Brazil.

${ }^{3,4}$ Graduandos de Medicina. Universidade Federal de Alagoas/UFAL. Maceió (AL), Brazil.

${ }^{5}$ Doutora. Universidade Federal de Alagoas/UFAL. Maceió (AL), Brazil.
}

Rev. Port. SaúdeSociedade. 2019;4(1): 961 - 974 
quantitative, cross-sectional and analytical study conducted with 101 PMMB doctors in Alagoas. The study was conducted in Maceió, in a non-probabilistic convenience sample, in 2017, at the time of the local-regional meeting of PMMB. A questionnaire was composed of ten multiple-choice questions addressing the topic of prenatal care in PC. The data was analyzed in the Epi Info program by statistical measures of frequency and chi-square. The issues were divided by themes: two addressing Gestational Diabetes Mellitus; two on maternal morbidity and mortality; three with respect to imaging tests in obstetrics and fetal vitality and three on complementary exams in prenatal care. Results: of the 101 physicians, $33.3 \%$ were Brazilian and $66.7 \%$ were foreign; $62.7 \%$ were women. Brazilian professionals obtained a higher average of hits compared to foreigners, $61.67 \%$ against $42.53 \%$, with the overall average being $49 \%$. Conclusion: this may suggest a discrepancy in the direction of educational models, with a better focus on prenatal care by Brazilian colleges.

Descriptors: Prenatal Care; Continuing Education; Primary Health Care; Doctors.

\section{RESUMEN}

Objetivo: evaluar el conocimiento sobre prenatal en la Atención Básica $(A B)$ entre médicos que actúan en el Programa Mais Médicos para o Brasil (PMMB). Método: se trata de un estudio cuantitativo, transversal y analítico, realizado con 101 médicos del PMMB en Alagoas. El estudio fue realizado en Maceió, en muestra por conveniencia, no probabilística, en 2017, en el momento del encuentro locorregional del PMMB. Se aplicó un cuestionario compuesto por diez preguntas de múltiple elección, abordando el tema del prenatal en la $A B$. Los datos fueron analizados en el programa Epi Info a través de medidas estadísticas de frecuencia y chi-cuadrado. Las preguntas fueron divididas por temas: dos abordando Diabetes Mellitus Gestacional; dos sobre morbimortalidad materna; tres a respeto de exámenes de imagen en obstetricia y vitalidad fetal y tres sobre exámenes complementarios en el prenatal. Resultados: de los 101 médicos, $33,3 \%$ eran brasileños y $66,7 \%$ extranjeros; el $62,7 \%$ eran mujeres. Los profesionales brasileños obtuvieron una media de aciertos superior en relación a los extranjeros, $61,67 \%$ contra $42,53 \%$, siendo la media general el $49 \%$. Conclusión: esto puede sugerir una discrepancia en el direccionamiento de los modelos educativos, con un mejor enfoque en el prenatal por parte de las facultades brasileñas.

Descriptores: Cuidado Prenatal; Educación Continuada; Atención Primaria a la Salud; Médicos.

INTRODUÇÃO

O Programa Mais Médicos no Brasil (PMMB) veio suprir a deficiência de lotação de médicos em locais carentes e distantes dos grandes centros. 0 programa foi criado por meio da Lei no 12.871, de 22 de outubro de 2013, com a finalidade de formar recursos humanos na área médica para o Sistema Único de Saúde (SUS), com os seguintes objetivos: diminuir a carência de médicos nas regiões prioritárias para o SUS; aprimorar a formação médica no país; ampliar a inserção do médico em formação nas unidades de atendimento do SUS; fortalecer a política de educação permanente; promover a troca de 
conhecimentos e aperfeiçoar médicos para a atuação nas políticas públicas de saúde. ${ }^{1}$

Em Alagoas, o programa foi implantado, também, em 2013, com a adesão da Universidade Federal de Alagoas (UFAL) e da Secretaria Estadual de Alagoas (SESAU), sendo esta última a coordenadora do processo. ${ }^{1}$

O PMMB adota as ações de reordenação da oferta de cursos de Medicina e de vagas para a residência médica, priorizando as regiões de saúde com a menor relação de vagas e médicos por habitante e com uma estrutura de serviços de saúde em condições de ofertar um campo de prática suficiente e de qualidade para os alunos. O programa tem, como metas, ainda, o estabelecimento de novos parâmetros para a formação médica no país e a promoção da saúde nas regiões prioritárias do SUS, com o aperfeiçoamento de médicos na área de Atenção Básica em Saúde, por meio da integração ensino-serviço, inclusive, por meio do intercâmbio internacional. ${ }^{1}$

A Atenção Básica $(A B)$ é um conjunto de ações de saúde, individual e coletiva, que abarca a promoção e a proteção da saúde, com a prevenção de agravos, diagnóstico, tratamento, reabilitação e a busca da conservação da saúde. A Estratégia de Saúde da Família (ESF) busca reorganizar a Atenção Básica no Brasil, seguindo os preceitos do SUS e substituindo os princípios da rede de $A B$ tradicional nos locais em que atuam as Equipes Saúde da Família. A ação deste programa tem, como metas, cadastrar os domicílios e diagnosticar a situação de saúde dos usuários para, então, realizar as ações dirigidas aos problemas de saúde. ${ }^{2}$ Entre as ações prioritárias da ESF, está o acompanhamento da gestação, particularmente, nas ações do pré-natal. A literatura aponta a proteção que a assistência adequada pré-natal oferece à saúde materna e neonatal, abrangendo as ações direcionadas à redução do risco e da severidade da morbimortalidade para o binômio mãe-filho. ${ }^{3}$

A gestação caracteriza-se por um período especial na vida da mulher, que exige a realização adequada do pré-natal. Este deve ser de qualidade e humanizado, incluindo ações preventivas e de promoção da saúde, bem como o diagnóstico e o tratamento adequados das doenças ou problemas que acontecerem durante esse período. ${ }^{3}$

No Brasil, verifica-se "o aumento do número de consultas de pré-natal por mulher que realiza o parto no SUS, partindo de 1,2 consultas por parto em 1995 para 5,1 consultas por parto em 2003" (SIA-Datasus e AIH-Datasus, 2004). 
Todavia, apesar dessa ampliação, a análise dos dados mostra, ainda, o deficit na qualidade dessa assistência, o que se evidencia pelas morbidades ainda presentes no período, como a sífilis congênita e hipertensão arterial, levando à mortalidade materna. ${ }^{4}$

Embora seja um fenômeno fisiológico que, geralmente, evolui sem intercorrências, a gestação demanda cuidados especiais no pré-natal, que deve acolher e acompanhar a mulher. Tem-se observado, todavia, que as consultas médicas de pré-natal no SUS são realizadas de forma rápida, levando a diagnósticos não adequados de possíveis anormalidades que passam despercebidas, impossibilitando, também, às mulheres, a manifestação adequada das suas queixas e dúvidas sobre a gravidez. ${ }^{4}$

As melhorias observadas na assistência obstétrica em relação ao acompanhamento e ao controle glicêmico durante a gestação levaram à ativa diminuição da morbimortalidade perinatal associada ao diabetes na gravidez, todavia, ainda ocorrem complicações neonatais, como membrana hialina, macrossomia, hipocalcemia, hiperbilirrubinemia, policitemia e hipomagnesemia em recém-nascidos de mães diabéticas. Não obstante, as malformações congênitas superam, em quase três vezes, aquelas observadas na população geral e representam a principal causa de mortalidade perinatal. ${ }^{2-6}$

O princípio básico utilizado pela dopplerfluxometria é o efeito Doppler. O Doppler contínuo é usado na propedêutica obstétrica para a ausculta dos batimentos cardiofetais e para a avaliação do fluxo sanguíneo nos vasos periféricos, sendo uma importante ferramenta na determinação do bem-estar fetal. A análise espectral Doppler fornece dados qualitativos (perfil de velocidades de determinado fluxo) e quantitativos (frequências sistólica e diastólica). $O$ aprimoramento da tecnologia permitiu expandir o âmbito da investigação às fases precoces da gestação e alargá-lo ao território venoso, incluindo o estudo do duto venoso. Assim, o uso da ultrassonografia e a popularização das suas indicações proporcionam a vigilância contínua essencial durante a gravidez. ${ }^{7}$

Entende-se, então, que o acompanhamento adequado à gestante resulta em benefícios para a mãe, feto e bebê, e que a detecção e o tratamento de prováveis morbidades diminuem a incidência do baixo peso ao nascer e da prematuridade, além de promoverem o incentivo ao aleitamento materno. A qualidade da assistência à gestante deve observar, entre outros fatores, o 
número de consultas e a idade gestacional de início do pré-natal, incorporando a atenção integral, destacando-se as ações educativas. ${ }^{8}$

Tornam-se importantes, portanto, o acompanhamento e a capacitação dos profissionais médicos para a assistência adequada, investindo na sua formação e educação permanente.

\section{MÉTODOS}

Trata-se de um estudo quantitativo, transversal e analítico, realizado com médicos que atuam no Programa Mais Médicos em Alagoas, por meio de uma amostra por conveniência não probabilística.

O estudo foi realizado em Maceió, em 2017, em um momento de reunião locorregional do PMMB, com a aplicação de um questionário composto por dez questões sobre o pré-natal na atenção básica. Os temas das questões indagadas no instrumento foram o DiabetesMellitusGestacional (DMG), a morbimortalidade materna, os exames de imagem em Obstetrícia e a solicitação de exames complementares no pré-natal.

O instrumento foi aplicado pelo supervisor dos médicos em grupos, divididos por municípios, em um total de 26 supervisores, que realizaram a correção dos instrumentos.

Os dados foram analisados no programa Epi Infopor meio de medidas estatísticas de frequência e qui-quadrado. Para a apresentação e a análise dos resultados, além do número de acertos por questão, as respostas dos sujeitos foram somadas de acordo com os temas pesquisados.

\section{RESULTS}

Foram estudados 101 médicos, sendo 33,3\% brasileiros e 66,7\% estrangeiros. Do total, $62,7 \%$ dos indivíduos do estudo eram mulheres. 0 total de testes foi de 105, contudo, três testes precisaram ser excluídos por não possuírem identificação, impossibilitando classificar os pesquisados quanto ao gênero e à nacionalidade. Outro teste foi descartado pois o participante não respondeu ao teste no tempo estabelecido previamente.

Apresenta-se o valor de acertos e erros por questão, sem divisão por sexo e nacionalidade (Tabela 1 ). As questões estão apresentadas por temas, sendo que

Rev. Port. SaúdeSociedade. 2019;4(1): 961 - 974 
as perguntas dois e dez abordam o Diabetes Mellitus Gestacional; a um e a nove, a morbimortalidade materna; os itens três, quatro e cinco, os exames de imagem em Obstetrícia e vitalidade fetal, e as questões seis, sete e oito, os exames complementares no pré-natal.

\section{Tabela 1. Distribuição das questões e número de acertos e erros por questões indagadas.}

\begin{tabular}{lccccc}
\hline Questão & Acertos & \% & Erros & \% & Total \\
\hline Q 1 & 98 & 97,03 & 3 & 2,97 & 101 \\
Q 2 & 41 & 40,59 & 60 & 59,41 & 101 \\
Q 3 & 78 & 77,23 & 23 & 22,77 & 101 \\
Q 4 & 9 & 8,91 & 92 & 91,09 & 101 \\
Q 5 & 68 & 67,33 & 33 & 32,67 & 101 \\
Q 6 & 47 & 46,53 & 54 & 53,47 & 101 \\
Q 7 & 28 & 27,72 & 73 & 72,28 & 101 \\
Q 8 & 29 & 28,71 & 72 & 71,29 & 101 \\
Q 9 & 65 & 64,36 & 36 & 35,64 & 101 \\
Q 10 & 32 & 31,68 & 69 & 68,32 & 101 \\
\hline
\end{tabular}

Nota: O algarismo após representa a sua ordem no questionário.

A discriminação por temas está apresentada na tabela 2. Usaram-se siglas para uma melhor visualização: DMG, para Diabetes Mellitus Gestacional; M.M., para morbimortalidade materna; E.I., para exames de imagem em Obstetrícia e vitalidade fetale E.C., para exames complementares no pré-natal.

Tabela 2. Distribuição das questões por tema e número de acertos e erros por temas abordados.

\begin{tabular}{|c|c|c|}
\hline Tema & Acertos\% & Erros \% \\
\hline DMG & 36,14 & 63,86 \\
\hline M. M & 80,69 & 19,31 \\
\hline E. I & 51,15 & 48,85 \\
\hline E. C. & 34,32 & 65,68 \\
\hline
\end{tabular}

O desempenho, dividido pelas nacionalidades envolvidas, é encontrado na tabela 3, onde são descritos o total de acertos, as respectivas médias do grupo envolvido e as pontuações mínimas e máximas realizadas pelos médicos submetidos à pesquisa. Observa-se a maior média de acertos nos pesquisados brasileiros (61,76\%), quando comparados aos estrangeiros (42,53\%). Além disso, a pontuação máxima foi encontrada entre os integrantes nacionais $(9,00$ contra 7,00), e a menor pontuação está no grupo dos participantes estrangeiros $(0,00$ contra 3,00$)$.

Rev. Port. SaúdeSociedade. 2019;4(1): 961 - 974 
Consecutivamente, foi realizada a análise dos dados encontrados por testes estatísticos, objetivando confirmar o valor estatístico da diferença de acertos entre brasileiros e estrangeiros. O teste $\mathrm{T}$ de Student demonstrou uma igualdade de variâncias entre as amostras estudadas, dentro do intervalo de confiança (IC 95\%). A análise de variância (ANOVA) encontrou um valor de $\mathrm{p}=$ 0,000, que apresentou o mesmo resultado no teste de Kruskal-Wallis. O quiquadrado de Bartlett encontrou um valor de $p=0,19248$, nesse caso, o valor de p maior que $5 \%$ indica uma análise de variância (ANOVA) apropriada, o que corrobora o resultado de uma variância homogênea. Dessa forma, encontrou-se uma significância estatística para a maior pontuação de brasileiros em relação aos participantes de demais nacionalidades.

Tabela 3. Número de acertos e erros nas questões por nacionalidade.

\begin{tabular}{lccccc}
\hline \multicolumn{1}{c}{ Grupo } & Participantes & Acertos* & Média (\%) & Máximo & Mínimo \\
\hline Estrangeiros & 67 & 285 & 42,53 & 7,00 & 0,00 \\
Brasileiros & 34 & 210 & 61,76 & 9,00 & 3,00 \\
\hline Total & $\mathbf{1 0 1}$ & $\mathbf{4 9 5}$ & $\mathbf{4 9 , 0 0}$ & $\mathbf{9 , 0 0}$ & $\mathbf{0 , 0 0}$ \\
\hline
\end{tabular}

\section{DISCUSSION}

A educação médica no Brasil é, historicamente, um assunto de amplo debate. Desta forma, é, também, sujeita a constantes alterações e remodelagens, em diferentes níveis. Entre estas, talvez a mais relevante da última década tenha sido a instituição das Diretrizes Curriculares Nacionais do Curso de Graduação em Medicina, no ano de 2014, pelo Conselho Nacional de Educação. ${ }^{9}$ Este documento cita, em seu artigo 30: "O graduado em Medicina terá formação geral, humanista, crítica, reflexiva e ética, com capacidade para atuar nos diferentes níveis de atenção à saúde $[\ldots]^{\prime \prime 9}$, determinando, assim, que os cursos médicos no Brasil têm, como objetivo primordial, a formação de profissionais generalistas, em oposição à especialização precoce.

Este fato é reforçado no artigo $24^{\circ}$ do documento, que estabelece as normas acerca do estágio obrigatório do curso médico: nos parágrafos três e quatro, determina-se que $30 \%$ da carga horária do internato médico deve ser dedicada à atenção básica e aos Serviços de Urgência e Emergência do SUS, com aquela predominando sobre estes ${ }^{9}$. Fica claro, portanto, que a formação de um profissional médico generalista, plenamente capacitado para o exercício da 
Atenção Básica à Saúde, deve ser a prioridade das graduações de Medicina do país.

Idealmente, a porta de entrada da gestante no sistema de saúde para o acompanhamento pré-natal é a Unidade Básica de Saúde (UBS). A equipe multidisciplinar deve ser capaz de oferecer, a tais pacientes, um acompanhamento contínuo, mesmo antes de a gravidez se iniciar, com aconselhamentos pré-concepcionais e a detecção precoce da gestação. Uma vez confirmada a gravidez, deve-se instituir o acompanhamento pré-natal da gestante o mais cedo possível. As pacientes de baixo risco podem ter o seu prénatal conduzido inteiramente na atenção básica pelo médico generalista, ao passo que as de alto risco devem ser reconhecidas precocemente e encaminhadas ao centro de referência aonde as demais consultas serão realizadas. ${ }^{10}$ Assim, evidencia-se que o papel do médico da atenção básica é primordial para o funcionamento de todos os níveis da rede de atenção à gestante.

Ao saber disso, é visível a necessidade de que o médico generalista domine, ao menos, os conhecimentos de Obstetrícia necessários para a condução das gestações de baixo risco e a identificação dos casos de alto risco. É urgente, portanto, que os cursos de Medicina do país promovam o aprendizado adequado destas competências e que estes critérios se transfiram para os profissionais estrangeiros que atuam na atenção básica no Brasil.

Ao considerar que o pré-natal é inerente à atenção básica e que esta pode ser exercida por médicos sem qualquer formação além da graduação, a análise do conhecimento dos generalistas acerca do tema é um importante dado na avaliação da educação médica no Brasil e do processo de permissão da atividade de profissionais estrangeiros. Uma análise individual das questões aplicadas neste estudo permitiu que se identificassem os assuntos com a menor porcentagem de acerto, o que pode representar uma abordagem insatisfatória por parte dos cursos de graduação, além de orientar programas de educação continuada para os profissionais da atenção básica.

Foi realizada uma pesquisa sistemática nas bases de dados LILACS e SciELO, além dos periódicos CAPES, com o intuito de se comparar este estudo, e os seus resultados, a pesquisas anteriores. Constatou-se que não há trabalhos prévios envolvendo a pesquisa sobre a adequação dos conhecimentos em prénatal na atenção básica por parte de médicos participantes do Programa Mais 
Médicos no Brasil. Assim, mostraram-se relevantes a discussão dos temas abordados no instrumento de pesquisa e o desempenho do grupo-alvo.

O Diabetes MellitusGestacional (DMG) tem, por definição, a intolerância aos carboidratos, que teve início na gestação atual e não se enquadra nos critérios de Diabetes Mellitusfranco. ${ }^{11}$ Trata-se de uma das patologias mais comuns da gestação, sendo a principal alteração metabólica nesse período, com uma prevalência entre 3\% e 25\%, dependendo dos critérios diagnósticos, da etnia e da população observada. ${ }^{11,12}$

O seu rastreamento, abordado nos quesitos dois e dez, é de fundamental importância para a prevenção de eventos adversos, tanto maternos quanto fetais, já que o DMG está associado às maiores morbidade e mortalidade perinatais. ${ }^{12}$ A principal complicação fetal é a macrossomia, que se associa à obesidade na infância e ao acréscimo no risco de síndrome metabólica na vida adulta. ${ }^{13}$ Além disso, a macrossomia fetal leva a um aumento no número de partos por via cesariana e das suas consequências, como um maior tempo de internação, o risco de hemorragias e as infecções puerperais. ${ }^{12,13}$

Apesar da conhecida importância do rastreamento do DMG, não há um consenso sobre como este deve ser feito. ${ }^{11-3}$ Entretanto, a maioria das referências cita que o screeningdeve ocorrer entre 24 e 28 semanas, ${ }^{11-4}$ o que foi arguido no $10^{\circ}$ quesito, período em que a doença se manifesta e há tempo para que a instituição de medidas terapêuticas apresente resultados. ${ }^{13}$ Entre algumas referências e o que é adotado no setor, o rastreamento é considerado positivo quando há uma glicemia de jejum igual ou superior a $85 \mathrm{mg} / \mathrm{dL}$, associada a um fator de risco, ou pelo Teste Oral de Tolerância à Glicose (TOTG), simplificado, em uma hora, com valor maior ou igual a $140 \mathrm{mg} / \mathrm{DI} .^{13,14} \mathrm{~A}$ despeito da relevância do tema em meio à atenção básica, observou-se um dos menores percentuais de acerto envolvendo este assunto, já que apenas 36,14\% dos indivíduos tiveram êxito.

A mortalidade materna é considerada um problema de saúde pública, principalmente, em países não desenvolvidos, que concentram 99\% dos óbitos maternos. No país, a hipertensão lidera como a sua causa, fato que se repete na região Nordeste, seguida das hemorragias e infecções. ${ }^{15}$ Aqui, observou-se o melhor desempenho dos médicos arguidos, onde houve $80,69 \%$ de sucesso nas respostas sobre o tema.

Rev. Port. SaúdeSociedade. 2019;4(1): 961 - 974 
A doença hipertensiva específica da gestação (DHEG), abordada na questão nove, é uma das complicações mais frequentes da gravidez, com apresentações variáveis, partindo de casos leves de hipertensão gestacional, passando pela pré-eclâmpsia, e chegando a formas graves, como a eclâmpsia e a síndrome HELLP. ${ }^{16}$ A DHEG é uma entidade exclusiva da segunda metade da gestação, com rara ocorrência antes das 20 semanas de gestação. ${ }^{17}$

A hemorragia puerperal está entre as principais causas de morte no mundo, sendo responsável, aproximadamente, por $25 \%$ de todas as mortes maternas. Pode suceder tanto o parto vaginal, com uma incidência entre $4 \%$ e $8 \%$, quanto o parto cesáreo, ocorrendo em cerca de $6 \%$ dos casos. 0 sangramento pode levar a várias complicações relacionadas com o pior desfecho, como o choque hipovolêmico, as coagulopatias, a insuficiência renal, a síndrome de Sheehan e a síndrome do desconforto respiratório do adulto. ${ }^{16}$

As infecções maternas são a terceira causa de mortalidade durante o intervalo gestação-puerpério. Os quadros infecciosos podem ser divididos em não obstétricos e obstétricos. Entre os não obstétricos, os descritos como principais relacionados à sepse são a infecção do trato urinário, a pneumonia adquirida na comunidade, a apendicite, a colecistite, o HIV e a malária. Já no grupo de sítio obstétrico, estão a endometrite, a corioamnionite, a infecção de episiotomia, a infecção de parede ou uterina pós-cesárea, o aborto infectado, além de outros de menor frequência. ${ }^{16,18}$ O quesito um, que versa sobre tal assunto, é onde se encontrou o maior percentual de acertos, 97,03\%, o que pode evidenciar a competência dos integrantes, tanto estrangeiros quanto brasileiros, acerca do abordado.

Os exames de imagem em Obstetrícia são ferramentas utilizadas para a avaliação da idade gestacional, morfologia fetal, placenta e os seus anexos, líquido amniótico e vitalidade fetal. Houve um resultado médio de sucesso nas questões que envolvem o uso dessa propedêutica, com um resultado de $51,15 \%$ dos envolvidos respondendo corretamente ao que se pediu.

O exame ultrassonográfico demonstra uma grande eficácia para a datação da idade gestacional e apresenta os resultados mais precisos quando feito no primeiro trimestre. A avaliação da morfologia fetal do primeiro trimestre é utilizada para rastrear as malformações fetais, como cromossomopatias, cardiopatias e síndromes genéticas. No segundo trimestre, o exame morfológico deve ser realizado entre 20 e 24 semanas. Já no terceiro trimestre, outras 
estruturas podem ser analisadas, como a placenta e o líquido amniótico. ${ }^{16,19} \mathrm{Em}$ uma revisão sistemática, concluiu-se que a utilização da ultrassonografia de rotina na assistência pré-natal apresenta benefícios, contudo, o tema continua controverso no que diz respeito à redução da mortalidade materna ou neonatal, em gestações de risco habitual. ${ }^{16}$

A vitalidade fetal é aferida, em uma das suas formas mais antigas, pela vigilância da percepção dos movimentos fetais, ${ }^{20}$ além do uso do sonar obstétrico, nas consultas de rotina pré-natal. Os métodos de propedêutica armada, que são a dopplervelocimetria, a cardiotocografia e o estudo biofísico fetal, não têm indicação de realização na assistência pré-natal de baixo risco, sendo reservados para as condições em que exista a possibilidade do comprometimento da oxigenação fetal. ${ }^{16,21}$ A dopplervelocimetria é o principal método que mensura as velocidades do fluxo sanguíneo nos vasos da circulação materna e fetal. ${ }^{21}$ Apesar da taxa de acertos razoável como um todo no conhecimento sobre exames de imagem e vitalidade fetal, a quarta questão, que trata da vitalidade, é onde se registra o pior desempenho por parte dos envolvidos, com um índice de acerto de apenas 8,91\%.

Por fim, avaliou-se o desempenho em itens que envolviam, principalmente, o uso de exames laboratoriais no pré-natal, revelando-se o pior resultado geral de um tema como um todo, onde houve apenas 34,32\% de sucesso nos quesitos arguidos. Tais exames são de fundamental importância, sendo utilizados no rastreio e prevenção de doenças que trazem prejuízos para a gestante e o feto. $\mathrm{Na}$ atenção básica da rede do SUS, o Ministério da Saúde elenca os exames complementares que devem ser solicitados no pré-natal de risco habitual, dividindo-os pelo trimestre em que a gestação se encontra.

Na primeira consulta, ou no primeiro trimestre, solicitam-se o hemograma, a tipagem sanguínea e o fator Rh, o teste de Coombs indireto (se o Rh for negativo), a glicemia de jejum, as sorologias para sífilis, toxoplasmose, HIV e hepatite $B$, e o sumário de urina mais urocultura. A colpocitologia oncótica, 0 exame parasitológico de fezes e o exame de secreção vaginal devem ser realizados se houver indicação clínica. ${ }^{22}$

Durante o segundo trimestre, o Ministério da Saúde indica a realização do TOTG, se houver um fator de risco para DMG ou glicemia de jejum acima de $85 \mathrm{mg} / \mathrm{dl}$, preferencialmente, entre as 24 e 28 semanas. Se o Rh materno for negativo, deve-se realizar o teste de Coombs indireto. ${ }^{22}$

Rev. Port. SaúdeSociedade. 2019;4(1): 961 - 974 
O terceiro trimestre é o período em que se devem solicitar o hemograma, a glicemia de jejum, o teste de Coombs indireto (se o Rh for negativo), o VDRL, o exame anti-HIV, a sorologia para hepatite $B$ e o sumário de urina mais urocultura. A sorologia de toxoplasmose deve ser repetida se o IgG não for reagente. A partir da 37 a semana de gestação, é necessária a coleta de material para a bacterioscopia de secreção vaginal. ${ }^{22}$

A avaliação do desempenho no teste por nacionalidade mostrou que os profissionais formados no Brasil tiveram um índice de acertos, em média, 19 pontos percentuais mais elevado que os médicos estrangeiros avaliados. Isso pode sugerir uma discrepância no direcionamento dos modelos educacionais, com um melhor enfoque no pré-natal por parte das faculdades brasileiras, principalmente, após a reforma curricular no Brasil. ${ }^{9}$ Porém, é importante ressaltar que, ainda que estatisticamente fundamentada, esta conclusão está sujeita a diversas variáveis de caráter individual e coletivo que não foram, necessariamente, reconhecidas neste estudo, de forma que se demandaria a confirmação por outros trabalhos similares.

\section{CONCLUSION}

Os resultados encontrados permitem concluir que, nesta amostra, os médicos formados no Brasil apresentaram um melhor desempenho no teste, ao se avaliar o índice de acertos por questão. O resultado associado às questões sobre DMG e sobre exames complementares solicitados no pré-natal foi inferior ao observado em outros temas, o que pode sugerir deficiências específicas nesses temas, dado valioso para a avaliação da aprendizagem do conteúdo e da necessidade do planejamento de educação continuada para os médicos da atenção básica.

Finalmente, mesmo com o melhor desempenho, os médicos não alcançaram um patamar ideal, especialmente, levando em conta a importância do serviço em questão para a saúde pública do país. Isto evidencia a necessidade de melhor avaliar o ensino médico brasileiro, reestruturando os aspectos necessários para um melhor preparo prático dos egressos para o exercício da profissão na atenção básica.

\section{REFERENCES}

Rev. Port. SaúdeSociedade. 2019;4(1): 961 - 974 
1. Lei no 12.871 , de 22 de outubro de 2013. Institui o Programa Mais Médicos, altera as Leis no 8.745, de 9 de dezembro de 1993, e no 6.932, de 7 de julho de 1981, e dá outras providências. Diário Oficial da União [Internet]. 2013 [cited 2018 Oct 22].Availablefrom: http://maismedicos.gov.br/legislacao. Acesso em: 10 maio 2017.

2. Ministério da Saúde (BR), Secretaria de Atenção à Saúde, Departamento de Atenção Básica. Política nacional de atenção básica [Internet]. Brasília: Ministério da Saúde;2006 [cited 2018 Aug 10].Availablefrom: http://189.28.128.100/dab/docs/publicacoes/geral/pnab.pdf

3. Líbera BD, Saunders C, Santos MMAS, Rimes KA, Brito FRSS, Baião MR. Evaluationofprenatalassistance in the point ofviewofpuerperasandhetalthcareprofessionals. Ciênc Saúde Colet. 2011; 16(12):4855-64. Doi:http://dx.doi.org/10.1590/S1413-81232011001300034

4. Ministério da Saúde (BR), Secretaria de Atenção à Saúde, Departamento de Ações Programáticas Estratégicas, Área Técnica de Saúde da Mulher. Pré-natal e Puerpério: atenção qualificada e humanizada - manual técnico [Internet]. Brasília: Ministério da Saúde;2005 [cited 2018 Aug 6].Availablefrom: http://bvsms.saude.gov.br/bvs/publicacoes/manual_pre_natal_puerperio_3ed.pdf

5. Landerdahl MC, Ressel LB, Martins FB, Cabral FB, Gonçalves MO. Women'sPerceptionAboutPre-ConceptionAttention in a Basic Health Unit. Esc Anna NeryRevEnferm [Internet]. 2007 Mar [cited 2018 Aug 5]; 11(1):10511.Availablefrom: https://www.redalyc.org/articulo.oa?id=127715305015

6. Mauad Filho F, Dias CC, Meirelles RS, Cunha SP, Nogueira A, Duarte G. Diabetes e gravidez: aspectos clínicos e perinatais. Revbrasginecol obstet. 1998 May; 20(4):193-8. Doi:http://dx.doi.org/10.1590/S0100-7203199800040000

7. Murta CGV, Batistuta PN, Cunha Filho JS. Doppler velocimetry in thestudyofthe fetal circulation: review onsafety. Radiol bras. 2002 Nov/Dec; 35(6):365-70.Doi: http://dx.doi.org/10.1590/S0100-39842002000600010

8. Silva EP, Lima RT, Osório MM. Impactofeducationalstrategies in lowriskprenatalcare: systematic review ofrandomizedclinicaltrials. Ciênc saúde coletiva. 2016 Sept; 21(9):2935-48. Doi:http://dx.doi.org/10.1590/141381232015219.01602015

9. Ministério da Educação (BR), Conselho Nacional de Educação. Resolução no 3, de 20 de junho de 2014. Institui Diretrizes Curriculares Nacionais do Curso de Graduação em Medicina e dá outras providências [Internet].Brasília: Ministério da Educação;2014 [cited 2018 Aug 9]. Availablefrom:https://abmes.org.br/legislacoes/detalhe/1609

10. Ministério da Saúde (BR), Secretaria de Atenção à Saúde, Departamento de Atenção Básica. Atenção ao pré-natal de baixo risco [Internet]. Brasília: Ministério da Saúde;2012 [cited 2018 Sept09]. Availablefrom:http://bvsms.saude.gov.br/bvs/publicacoes/cadernos_atencao_basi ca_32_prenatal.pdf

11. MilechA. organizadores. Diretrizes da Sociedade Brasileira de Diabetes(20152016). São Paulo: A.C. Farmacêutica; 2016.

12. Mattar R, Zamarian ACP, Caetano ACR, Torloni MR, Negrato CA. Whatshouldbethescreeninganddiagnosticstrategies for gestational diabetes? 
Femina [Internet]. 2011 Jan [cited 2018 Aug 6]; 39(1):30-4.Availablefrom: http://files.bvs.br/upload/S/0100-7254/2011/v39n1/a2385.pdf

13. Bolognani CV, Souza SS, Calderon IMP. Gestational diabetes mellitus - focuson new diagnosticcriteria. Com Ciênc Saúde [Internet]. 2011 [cited 2018 Apr 9]; 22(1):31-42.Availablefrom:

http://bvsms.saude.gov.br/bvs/artigos/diabetes_mellitus_gestacional.pdf

14. Jacob TA, Soares LR, Santos MR, Santos LR, Santos ER, Torres GC, et al. Gestational Diabetes Mellitus: A Literature Review. Braz J SurgClin Res [Internet]. 2014 Mar/May [cited 2018 Apr 9]; 6(2):33-7.Availablefrom: https://www.mastereditora.com.br/periodico/20140331_212133.pdf

15. Morse ML, Fonseca SC, Barbosa MD, Calil MB, Eyer FPC. Maternal mortality in Brazil: whathasthescientificliteratureshown in thelast 30 years?Cad Saúde Pública. 2011 Apr; 27(4):623-38.Doi: http://dx.doi.org/10.1590/S0102$311 \times 2011000400002$

16. Zugaib, M. organizador. Obstetrícia. São Paulo: Manole;2011.

17. Novo JLVG, Gianini RJ. Eclampsia as a cause of maternal mortality. RevBrasSaúde Materno-Infantil. $2010 \quad$ Apr/June; $\quad 10(2): 209$ 17.Doi:http://dx.doi.org/10.1590/S1519-38292010000200008

18. Castro EO, Bortolotto MRFL, Zugaib M. Sepsisandsepticshockduringpregnancy: clinical management. RevBrasGinecol Obstet. 2008 Dec; 30(12):631-8. Doi:http://dx.doi.org/10.1590/S0100-72032008001200008

19. Meleti D, Caetano ACR, Nardozza LMM, Araujo Junior E, Moron AF. Does routineultrasound in lowriskpregnancycollaboratewiththereductionof maternal and neonatal mortality? Femina [Internet]. 2010 Aug [cited 2010 Sept19]; 38(8):4369. Availablefrom:http://files.bvs.br/upload/S/0100-7254/2010/v38n8/a1613.pdf

20. Nomura RMY, Ferreira MVC, Latif IOA, Francisco RPV, Zugaib M. Agreementbetween maternal perceptionof fetal movementsandvisualizationbyultrasound. RevBrasGinecolObstet. 2013 Feb; 35(2):55-9.Doi: http://dx.doi.org/10.1590/S0100-72032013000200003

21. Nomura RMY, Miyadahira S, Zugaib M. Antenatal fetal surveillance. Revista Brasileira de Ginecologia-Obstetrícia. 2009 Oct; 31(10):513-26. Doi:http://dx.doi.org/10.1590/S0100-72032009001000008

22. Ministério da Saúde (BR), Secretaria de Atenção à Saúde, Departamento de Atenção Básica. Atenção ao pré-natal de baixo risco [Internet]. Brasília: Ministério da Saúde;2012 [cited 2018 Apr 5]. Availablefrom: http://bvsms.saude.gov.br/bvs/publicacoes/cadernos_atencao_basica_32_prenat al.pdf 